\title{
Activation of DNA Damage Response and Cellular Senescence in Cardiac Fibroblasts Limit Cardiac Fibrosis After Myocardial Infarction
}

\author{
Masato Shibamoto, ${ }^{1 *}$ MD, Tomoaki Higo, ${ }^{1 *}$ MD, Atsuhiko T. Naito, ${ }^{2}$ MD, Akito Nakagawa, ${ }^{1}$ MD, \\ Tomokazu Sumida, ${ }^{3}$ MD, Katsuki Okada, ${ }^{1}$ MD, Taku Sakai, ${ }^{1}$ MD, Yuki Kuramoto, ${ }^{1}$ MD, \\ Toshihiro Yamaguchi, ${ }^{4} \mathrm{MD}$, Masamichi Ito, ${ }^{4} \mathrm{MD}$, Yuki Masumura, ${ }^{1} \mathrm{MD}$, Shuichirou Higo, ${ }^{1} \mathrm{MD}$, \\ Jong-Kook Lee, ${ }^{1,5}$ MD, Shungo Hikoso, ${ }^{1}$ MD, Issei Komuro, ${ }^{4}$ MD and Yasushi Sakata, ${ }^{1}$ MD
}

\begin{abstract}
Summary
Cardiac fibrosis plays an important role in cardiac remodeling after myocardial infarction (MI). The molecular mechanisms that promote cardiac fibrosis after MI are well studied; however, the mechanisms by which the progression of cardiac fibrosis becomes attenuated after MI remain poorly understood. Recent reports show the role of cellular senescence in limiting tissue fibrosis. In the present study, we tested whether cellular senescence of cardiac fibroblasts $(\mathrm{CFs})$ plays a role in attenuating the progression of cardiac fibrosis after MI. We found that the number of $\gamma \mathrm{H} 2 \mathrm{AX}$-positive $\mathrm{CFs}$ increased up to day 7 , whereas the number of proliferating $\mathrm{CFs}$ peaked at day 4 after MI. Senescent CFs were also observed at day 7, suggesting that attenuation of CF proliferation occurred simultaneously with the activation of the DNA damage response (DDR) system and the appearance of senescent CFs. We next cultured senescent CFs with non-senescent CFs and showed that senescent CFs suppressed proliferation of the surrounding non-senescent CFs in a juxtacrine manner. We also found that the blockade of DDR by Atm gene deletion sustained the proliferation of CFs and exacerbated the cardiac fibrosis at the early stage after MI. Our results indicate the role of DDR activation and cellular senescence in limiting cardiac fibrosis after MI. Regulation of cellular senescence in CFs may become one of the therapeutic strategies for preventing cardiac remodeling after MI.
\end{abstract}

Key words: Fibroblast senescence, Juxtacrine, Atm, Cardiac remodeling

(Int Heart J 2019; 60: 944-957)

$\mathrm{M}$ yocardial infarction (MI) is one of the major causes of chronic heart failure (CHF), which affects 26 million people worldwide. ${ }^{1)}$ Following MI, cardiac fibroblasts (CFs) in the infarct area become activated, proliferate, and produce an extracellular matrix to replace the necrotic tissue with fibrotic tissue. ${ }^{2,3}$ These processes are termed cardiac fibrosis. Cardiac fibrosis is necessary for maintaining tissue integrity after MI, but excess cardiac fibrosis leads to adverse cardiac remodeling and $\mathrm{CHF}^{4)}$ Although the mechanism by which CFs are activated and start proliferation is well studied, ${ }^{5-7)}$ how activated CFs return to a non-proliferative state after MI remains poorly understood.

Upon DNA damage, DNA damage response (DDR) signaling becomes activated to stop the cell cycle and repair damaged DNA. Ataxia telangiectasia mutated (ATM) is one of the key protein kinases that transduces the DDR signal to the effector proteins through phosphorylating its substrates, such as histone variant $\mathrm{H} 2 \mathrm{AX}$ and tumor suppressor $\mathrm{p} 53 .{ }^{8.9)}$ When the damage to the DNA is too severe to be repaired, DDR becomes persistent and triggers apoptotic cell death or stops the cell cycle permanently by inducing cellular senescence. ${ }^{10,11)}$ Recent reports suggest that activated hepatic fibroblasts become senescent after fibrotic liver injury and play a role in limiting tissue fibrosis both in a cell autonomous and a non-cell autonomous manner. ${ }^{12,13)}$

In the present study, we investigated the role of $\mathrm{CF}$ senescence in cardiac fibrosis after MI. We show that DDR is activated, and cellular senescence takes place in CFs after MI. We also show in vitro and in vivo evidence that senescent CFs suppress proliferation of surrounding

From the ${ }^{1}$ Department of Cardiovascular Medicine, Osaka University Graduate School of Medicine, Osaka, Japan, ${ }^{2}$ Department of Pharmacology, Faculty of Medicine, Toho University, Tokyo, Japan, ${ }^{3}$ Department of Neurology, Yale School of Medicine, New Haven, USA, ${ }^{4}$ Department of Cardiovascular Medicine, Graduate School of Medicine, The University of Tokyo, Tokyo, Japan and ${ }^{5}$ Department of Advanced Cardiovascular Regenerative Medicine, Osaka University Graduate School of Medicine, Osaka, Japan.

*These authors contributed equally to this work.

This study was supported by grants from JSPS KAKENHI (23689038), Agency for Medical Research and Development, AMED (JP17am0101122), SENSHIN Medical Research Foundation, The Cell Science Research Foundation (to A.T.N.), CREST, JST (to I.K.).

Address for correspondence: Atsuhiko T. Naito, MD, Department of Pharmacology, Faculty of Medicine, Toho University, 5-21-16 Omori-nishi, Tokyo, 143-8540, Japan. E-mail: atsuhiko.naito@med.toho-u.ac.jp

Received for publication December 12, 2018. Revised and accepted January 18, 2019.

Released in advance online on J-STAGE June 28, 2019.

doi: 10.1536/ihj.18-701

All rights reserved by the International Heart Journal Association. 
Table. Primers for PCR Genotyping

\begin{tabular}{lcc}
\hline Gene & Fw/Rev & Sequences \\
\hline Atm WT-allele $(147 \mathrm{bp})$ & Fw & GCTGCCATACTTGATCCATG \\
& Rev & TCCGAATTTGCAGGAGTTG \\
Atm mutant-allele $(280 \mathrm{bp})$ & Fw & CTTGGGTGGAGAGGCTATTC \\
& Rev & AGGTGAGATGACAGGAGATC \\
GFP $(650 \mathrm{bp})$ & Fw & GCCCACTAAGATAGCTCATTGACCTTGTCC \\
& Rev & CGCGGAACTCCATATATGGGCTATG \\
\hline
\end{tabular}

non-senescent CFs in a cell-cell, contact-dependent manner and play a role in attenuating the progression of cardiac fibrosis. Regulation of cellular senescence in CFs may become one of the therapeutic strategies for limiting cardiac fibrosis and preventing cardiac remodeling after MI.

\section{Methods}

Animal: C57BL/6J mice and fetal ICR mice were from CLEA Japan, Inc. Atm ${ }^{+/}$mice $^{14)}$ were from Jackson Laboratory (stock\#008536, stock name: B6.129S6-Atm ${ }^{\mathrm{tm} / \mathrm{Awb} / \mathrm{J}}$ ). The Atm wild-type (WT) and mutant alleles were detected by genotyping PCR according to the protocol from Jackson Laboratory. C57BL/6-Tg (CAG-EGFP) C14-Y01-FM 1310 sb mice, ${ }^{15)}$ in which EGFP expression was under the control of the CAG promoter, were from RIKEN Bioresource Center (BRC00267). The PCR primers are shown in the Table. All experiments were approved by the Institutional Animal Care and Use Committee of Osaka University (22-056) and carried out in accordance with its guidelines.

Myocardial infarction model: To generate a mouse model of MI, 8- to 12-week old male mice were anesthetized with $2.8 \%$ isoflurane inhalation under mechanical ventilation. The heart was exposed by a left thoracotomy followed by the ligation of left anterior descending coronary artery (LAD) using 8-0 nylon suture. ${ }^{16)}$ Myocardial ischemia was confirmed by the changes in the color of heart tissue and the motion of left ventricular (LV) wall. Transthoracic echocardiography was performed with Vevo 770 imaging system (Visualsonics, Inc.). M-mode echocardiographic image in a longitudinal view was used to measure the LV function and size.

Immunofluorescence: For immunofluorescent staining, the hearts were excised and immediately embedded in Tissue-Tek OCT compound (Sakura Finetek Japan). Cryostat sections at $4 \mu \mathrm{m}$ were fixed in acetone, washed in PBS, and incubated in either 5\% normal donkey serum (Sigma) or Mouse on Mouse basic kit (Vector Laboratories) to block nonspecific binding. Sections were then incubated with primary antibodies overnight. After washing with PBS, samples were incubated with fluorochromeconjugated secondary antibodies for 1 hour, and the nuclei were counterstained with TO-PRO-3 iodide 642/661 (1: 200) (Thermo Fisher Scientific). For the staining of cultured cells, cells were fixed with $4 \%$ paraformaldehyde (PFA) and permeabilized with $0.25 \%$ Triton-X100. Cells were then incubated with primary and secondary antibodies as described above. Images were obtained by the con- focal laser scanning microscopy LSM700 (Carl Zeiss), FSX100 (Olympus) or IN Cell Analyzer 6000 (GE Healthcare). The infarct area was distinguished visually. The border area was defined as the region within $100 \mu \mathrm{m}$ from the infarct area. The primary antibodies used were as follows: rabbit polyclonal anti-H2AX (phospho-Ser139) (1:500) (ab2893, Abcam), chicken polyclonal antivimentin (1:500) (ab24525, Abcam), mouse monoclonal anti-Histone H3 (phospho-Ser10) (1:500) (ab14955, Abcam), rabbit polyclonal anti-green fluorescent protein (GFP) (1:200) (ab6556, Abcam) and mouse monoclonal anti-alpha smooth muscle actin (1:250) (ab7817, Abcam). The secondary antibodies used were as follows: anti-rabbit IgG-Alexa 488 (1:200) (A-21206, Thermo Fisher Scientific), streptavidin-Alexa 488 (1:200) (S32354, Thermo Fisher Scientific), anti-mouse IgG-Alexa 594 (1:200) (A21203, Thermo Fisher Scientific), and anti-chicken IgYAlexa 594 (1:200) (703-585-155, Jackson ImmunoResearch).

Flow cytometry, FACS: Cell samples were prepared by sequentially digesting the minced heart tissue with dispase II (Wako) in PBS, followed with collagenase type 1 (Wako) in PBS containing 4\% fetal bovine serum (FBS, Biowest), $0.9 \mathrm{mM} \mathrm{CaCl}_{2}$ and $0.33 \mathrm{mM} \mathrm{MgCl}$. Cells were then pelleted, suspended in PBS containing 4\% FBS, and filtered through $40 \mu \mathrm{m}$ cell strainer (BD Falcon). ${ }^{17)}$ Nonspecific binding was blocked using anti-mouse CD16/32 Antibody (1:100) (101301, BioLegend). For fluorescentactivated cell sorting (FACS), cells were stained with antimouse CD31-BV421 (1:100) (562939, BD Biosciences), anti-mouse CD45-APC (1:50) (559864, BD Biosciences), anti-mouse Thy1-FITC (1:50) (553013, BD Biosciences), and anti-mouse PDGFR $\alpha$-PE (1:80) (135905, Biolegend). 7-aminoactinomysin D (1:100) (Thermo Fisher Scientific) was used for dead cell exclusion. Cell sorting was performed by BD FACSAria II. For flow cytometric analysis of $\gamma \mathrm{H} 2 \mathrm{AX}$, cells were fixed and permeabilized by using eBioscience $^{\mathrm{TM}}$ Intracellular Fixation \& Permeabilization Buffer Set (eBioscience) according to the manufacturer's instructions. Cells were then stained with anti-mouse CD 31-BV421 (1:100), anti-mouse CD45-APC (1:50), and anti-mouse H2AX (phospho-Ser139)-FITC ( $1 \mu \mathrm{L} / 1 \times 106$ cells) (613403, Biolegend), and counterstained with Fixable Viability Stain 510 (1:1,000) (BD Biosciences) for dead cell exclusion. Cells were analyzed by BD FACSAria II and Flo Jo software (Tree Star)

TUNEL assay: TdT-mediated dUTP nick end labeling (TUNEL) assay was performed by using in situ Apoptosis Detection Kit (Takara Bio Inc.) according to the manufacturer's instructions. Briefly, cryostat sections at $4 \mu \mathrm{m}$ or 
cells cultured on gelatin-coated coverslips were fixed with $3 \%$ PFA, permeabilized and incubated with TdT enzyme, including FITC-conjugated dUTP for 60 minutes at $37^{\circ} \mathrm{C}$. Nuclei were counterstained with TO-PRO-3 iodide 642/ 661 (1:200).

RNA analysis: Total RNA was extracted using the TRIzol Reagent (Life Technologies) or the PureLink RNA Mini Kit (Life Technologies) and treated with DNase using TURBO DNA-free Kit (Invitrogen) or PureLink DNase Set (Invitrogen), respectively. Reverse transcription was performed by using the SuperScript VILO cDNA Synthesis Kit (Invitrogen). Quantitative real-time PCR (qPCR) was performed by using THUNDERBIRD Probe qPCR Mix (Toyobo) with the target-specific primers and the matching probes designed by the Universal Probe Library System (Roche Applied Science). Relative expression levels of the target genes were calculated by comparative $\mathrm{Ct}$ method using $28 \mathrm{~S}$ ribosomal RNA as an internal control.

Isolation and culture of cardiac fibroblasts: CFs were isolated from the heart of mice embryo around embryonic day 15. Since few CFs can be obtained from a single embryo, we pooled the CFs from approximately 60 embryos. The heart was quickly removed and digested in $\mathrm{Ca}^{2+}$-free Hank's balanced salt solution (Cellgro) containing $100 \mu \mathrm{g}$ / $\mathrm{mL}$ trypsin (TRLSNK, Worthington Biochemical Corp.) and $70 \mathrm{U} / \mathrm{mL}$ collagenase (CLSPANK, Worthington Biochemical Corp.). Cells were then plated onto a tissue culture plate for 1 hour, and the supernatant containing cardiomyocytes was removed. Attached cells, containing mainly CFs, were cultured in Medium 199 (M 199) (Cellgro) containing 15\% FBS for an additional 24 hours, and the cells were thereafter cultured in Dulbecco's modified Eagle medium (Wako) containing 10\% FBS, $100 \mathrm{U} / \mathrm{mL}$ penicillin and $100 \mu \mathrm{g} / \mathrm{mL}$ streptomycin. We used 24-Well ThinCert $^{\mathrm{TM}}$ Cell Culture Inserts $(662630$, Greiner) for Boyden chamber system.

Cell proliferation assay: Proliferating CFs were labeled by culturing them in the presence of 5-bromo-2'deoxyuridine (BrdU) or 5-ethynyl-2'-deoxyuridine (EdU) $(10 \mu \mathrm{M})$ for 24 hours. BrdU-labeled cells were denatured with $2 \mathrm{M} \mathrm{HCl}$ for 10 minutes and incubated with rat monoclonal anti-BrdU (1:200) (ab6326, Abcam) followed by anti-rat IgG-Alexa 488 (1:200) (A-21208, Thermo Fisher Scientific). EdU-labeled cells were stained according to the manufacturer's protocol (Click-it Plus EdU Alexa Fluoro 594 Imaging Kit, Thermo Fisher Scientific). Nuclei were counterstained with TO-PRO-3 iodide 642/661 (1: 200) or NucBlue ${ }^{\circledR}$ Live ReadyProbes ${ }^{\circledR}$ Reagent (Thermo Fisher Scientific).

Western blot: Cultured CFs were homogenized and lysed with RIPA buffer with protease and phosphatase inhibitor cocktails for 30 minutes on ice. The cell lysate was centrifuged at $15,000 \mathrm{~g}$ for 20 minutes and the supernatant was used as whole-cell extract. For nuclear fractionation, heart tissue was homogenized and lysed in hypotonic buffer (10 $\mathrm{mM}$ HEPES, $1.5 \mathrm{mM} \mathrm{MgCl}, 10 \mathrm{mM} \mathrm{KCl}, 0.1 \mathrm{mM}$ DTT, and $0.05 \% \mathrm{NP}-40, \mathrm{pH} 7.9$ ) with protease and phosphatase inhibitor cocktails (respectively, Roche) for 10 minutes on ice. Nuclei were pelleted by centrifugation at $800 \mathrm{~g}$ for 10 minutes and lysed in RIPA buffer to obtain nuclear extracts. The protein samples were subjected to SDS-PAGE and transferred to nitrocellulose membranes (GE Healthcare). After blocking with 5\% skim milk, the membranes were incubated with mouse monoclonal anti-ATM antibody $(1: 10,000)$ (ab59541, Abcam), and rabbit monoclonal anti-GAPDH $(1: 10,000)$ (\#2118, Cell Signaling Technology) or rabbit polyclonal anti-Histone H3 antibody (1: 5,000) (H0164, Sigma), followed by horseradish peroxidase-conjugated anti-mouse or anti-rabbit IgG (715035-151 or 711-035-152, respectively, Jackson ImmunoResearch). The immunoreactive signal was detected with ECL Plus Western Blotting Detection System (GE Healthcare), and the images were obtained by ImageQuant LAS4000 (GE Healthcare).

Gene knockdown: For gene silencing, cultured CFs were transfected with pre-designed siRNAs against Atm (s62962, Ambion) using Lipofectamine RNAiMAX Transfection Reagent (13778150, Invitrogen) according to the manufacturer's instructions. A negative control duplex (Medium GC Duplex, 12935300, Invitrogen) was used as control. Oligonucleotide sequences of the siRNA against Atm were 5'-GGCUGUCAACUUCCGAAAAtt -3' (sense) and 5'-UUUUCGGAAGUUGACAGCCag -3' (antisense).

Histochemical staining: For histochemical staining, cryostat sections at $8 \mu \mathrm{m}$ were fixed in $2 \%$ PFA for three hours at $4^{\circ} \mathrm{C}$ and stained with hematoxylin and eosin (MUTO PURE CHEMICALS CO., LTD.), or with Trichrome Stain (Masson) Kit (Sigma) and Weigert's iron hematoxylin solution (Sigma), according to manufacturer's protocol. The LV \%fibrotic area was calculated as the ratio of the fibrotic area to the total area at the papillary muscle level and apex level using ImageJ software (National Institutes of Health, Bethesda, MD). Senescenceassociated beta-galactosidase (SA- $\beta$-gal) staining was performed using Senescence Detection Kit (Abcam). For the staining of heart tissue, cryostat sections at $8 \mu \mathrm{m}$ were fixed in $2 \%$ PFA for 3 hours at $4{ }^{\circ} \mathrm{C}$ and stained for 48 hours at $37^{\circ} \mathrm{C}$. The cell nuclei were stained by Nuclear Fast Red (Labvision). For the staining of cultured cells, cells were fixed in 2\% PFA for 15 minutes, and stained for 16 hours at $37^{\circ} \mathrm{C}$. The cell nuclei were stained by NucBlue ${ }^{\circledR}$ Live ReadyProbes ${ }^{\circledR}$ Reagent. Images were obtained by BZ-9000 (Keyence).

Statistical analysis: All data are presented as mean \pm s.e.m. Two-group comparison was performed by unpaired two-tailed Student's $t$-test or Mann-Whitney $U$-test. Multiple group comparison was performed by Dunnett test and Tukey-Kramer HSD test. Survival after MI operation was analyzed by Kaplan-Meier method and a two-group comparison was performed by Wilcoxon test. Statistical analyses were performed with JMP Pro 13.2.1 (SAS Institute Inc). Significant differences were defined as $P<0.05$.

\section{Results}

Activation of DDR and cellular senescence in cardiac fibroblasts after MI: To examine the role of DDR activation and cellular senescence in cardiac fibrosis after MI, we first analyzed the time course of DDR activation and the markers of cellular senescence in CFs. We found that the number of CFs positive for phosphorylated $\mathrm{H} 2 \mathrm{AX}(\gamma \mathrm{H}$ $2 \mathrm{AX}$ ), a marker that labels the cells with active DDR, in- 
creased from day 3 up to day 7 in the border and infarct area after MI (Figure 1A, B). Flow cytometric analysis also revealed that the fluorescence intensity of $\gamma \mathrm{H} 2 \mathrm{AX}$ in CFs increased 1.5-fold at day 7 after MI (Figure 1C, D). The expression level of Cdknla (p21) gene, a cyclindependent kinase inhibitor that is increased in senescent cells, was increased in $\mathrm{CD} 31^{-} \mathrm{CD} 45^{-}$Thy $1^{+}$and/or $\mathrm{CD} 31^{-}$ CD45-PDGFR $\alpha^{+} \mathrm{CFs}$ at day 7 after MI (Figure 1E). A number of $\mathrm{CFs}$ positive for senescence-associated betagalactosidase (SA- $\beta$-gal), which is the most wellestablished marker for cellular senescence, ${ }^{18)}$ were also observed in the border and the infarct area at day 7 after MI (Figure 1F). We then analyzed the time course of CF proliferation. The number of CFs positive for phosphorylated histone $\mathrm{H} 3$ ( $\mathrm{pHH} 3$ ), a marker for proliferating cells, also increased up to day 4 but decreased thereafter (Figure 2A, B). TUNEL-positive CFs were observed at only low levels and did not increase after MI (Figure 2C, D). Our observations show that the activation of DDR in CFs and the appearance of senescent CFs occurred simultaneously with deceleration of $\mathrm{CF}$ proliferation suggesting the strong relationship between these processes.

Establishment of an in vitro model of stress-induced senescence in CFs: To investigate the role of DDR activation and cellular senescence on the proliferation of CFs, we first established an in vitro model of senescent CFs by ionizing radiation (IR). ${ }^{1920)}$ The number of BrdU-positive CFs significantly decreased at the dose more than 2 Gy (Figure 3A, B). The number of $\gamma \mathrm{H} 2 \mathrm{AX}$-positive CFs increased (Figure 3C, D) and senescent CFs appeared at the dose more than 5 Gy (Figure 3E, F). There was a statistically significant increase in the number of TUNELpositive CFs at the dose of 10 Gy but not at 5 Gy (Figure $3 \mathrm{G}, \mathrm{H})$. These results collectively suggest that irradiation at the dose of 5 Gy activates DDR, impairs proliferative capacity, and induces cellular senescence without affecting cellular apoptosis in cultured CFs.

Senescent CFs suppressed the proliferation of the surrounding non-senescent CFs: Senescent cells alter the phenotype of surrounding non-senescent cells via paracrine or juxtacrine mechanisms. ${ }^{21,22)}$ We therefore examined the paracrine and/or juxtacrine effects of senescent CFs on the proliferative capacity of non-senescent CFs. We first examined the paracrine effect by using Boyden chamber system. ${ }^{23)}$ We cultured either irradiated or nonirradiated CFs in the upper chamber and co-cultured with CFs in the lower chamber for 72 hours. The proliferative capacity of the CFs in the lower chamber was assessed by incorporation of EdU for the last 24 hours (Figure 4A). Irradiation to the $\mathrm{CFs}$ in the upper chamber did not affect the percentage of EdU-positive CFs in the lower chamber, suggesting that senescent CFs do not affect the proliferative capacity of non-senescent $\mathrm{CFs}$ in a paracrine manner (Figure 4B, C). We next examined the juxtacrine effect of senescent $\mathrm{CFs}$ by culturing the non-labeled CFs, either irradiated or non-irradiated, with GFP-labeled CFs in a mixed culture and assessed the proliferative capacity of GFP-labeled CFs (Figure 5A). In this case, irradiated, senescent $\mathrm{CFs}$ significantly attenuated the proliferation of GFP-labeled CFs (Figure 5B, C) suggesting that senescent $\mathrm{CFs}$ suppress the proliferation of surrounding $\mathrm{CFs}$ in a juxtacrine manner (Figure 5D)

We also examined the involvement of ATM in DDR, cellular senescence and the juxtacrine effect of senescent cells on CF proliferation. ${ }^{24,25)}$ Knockdown of Atm gene by siRNA attenuated the activation of DDR (Figure 6A) and the appearance of senescent cells after irradiation (Figure 6B). Moreover, irradiated CFs with Atm gene knockdown showed a less suppressive effect on the proliferation of surrounding CFs (Figure 6C, D), suggesting that the juxtacrine effect of senescent $\mathrm{CFs}$ on the proliferation of surrounding non-senescent CFs is also mediated by ATM.

Cardiac fibrosis was exacerbated in ATM heterozygous mice: We finally investigated the role of ATM in cardiac fibrosis after MI. Heterozygous deletion of Atm inhibits persistent DDR activation in heart failure model mice. ${ }^{26}$ We therefore induced MI in ATM heterozygous mice $\left(\mathrm{Atm}^{+-}\right.$mice) and observed the effect of Atm gene deletion on $\mathrm{CF}$ proliferation, cellular senescence, and cardiac fibrosis after MI. Decreased expression of ATM was confirmed in the heart tissue and CFs of $\mathrm{Atm}^{+/-}$mice at both mRNA and protein level (Figure 7A, B). In $\mathrm{Atm}^{+/-}$mice, the number of pHH3-positive proliferating $\mathrm{CFs}$ was more observed (Figure 7C, D), whereas the number of SA- $\beta$-galpositive CFs was less observed (Figure 7E) at day 7 after MI compared with wild-type (WT) mice. Masson's trichrome staining showed that the fibrotic area at both mid and apical level of the heart was significantly increased at day 7 after MI (Figure 8A, B). It should be noted that the effect of heterozygous Atm gene deletion on cardiac fibrosis became comparable with WT mice at day 28 after MI (Figure 8A, B). In addition, LV function (Figure $8 \mathrm{C}$ ), the signs of heart failure (Figure 8D) and the mortality (Figure 8E) were also comparable between $\mathrm{Atm}^{+/}$and WT. These results suggest that ATM is involved in CF proliferation and cardiac fibrosis only at the early phase after MI, which may not affect the long-term outcome of MI, at least in mice.

\section{Discussion}

Excess activation and proliferation of CFs after $\mathrm{MI}$ lead to adverse cardiac remodeling and $\mathrm{CHF}^{2,3)}$ On the other hand, proper activation and proliferation of CFs after MI are required to maintain the strength of the myocardium and prevent cardiac rupture. ${ }^{4)}$ In a mouse model of MI, activation and proliferation of CFs are observed soon after coronary artery ligation. ${ }^{3)}$ In the present study, we found that the activation of DDR and the induction of cellular senescence occurred simultaneously in these proliferating CFs at the subsequent stage after coronary artery ligation. We also showed that the blockade of DDR effector ATM liberated CFs from cellular senescence and exacerbated cardiac fibrosis after MI, indicating that DDR activation and cellular senescence play critical roles in suppressing the proliferation of CFs. Recent reports show the involvement of cellular senescence in regulation of tissue fibrosis. ${ }^{12,13,27)}$ Upon fibrotic liver damage, hepatic stellate cells, resident fibroblasts in the liver tissue, become activated and proliferate to form liver fibrosis. Some of the activated stellate cells become senescent thereafter and senescent stellate cells in turn limit liver fibrosis. ${ }^{12)}$ A similar 
A
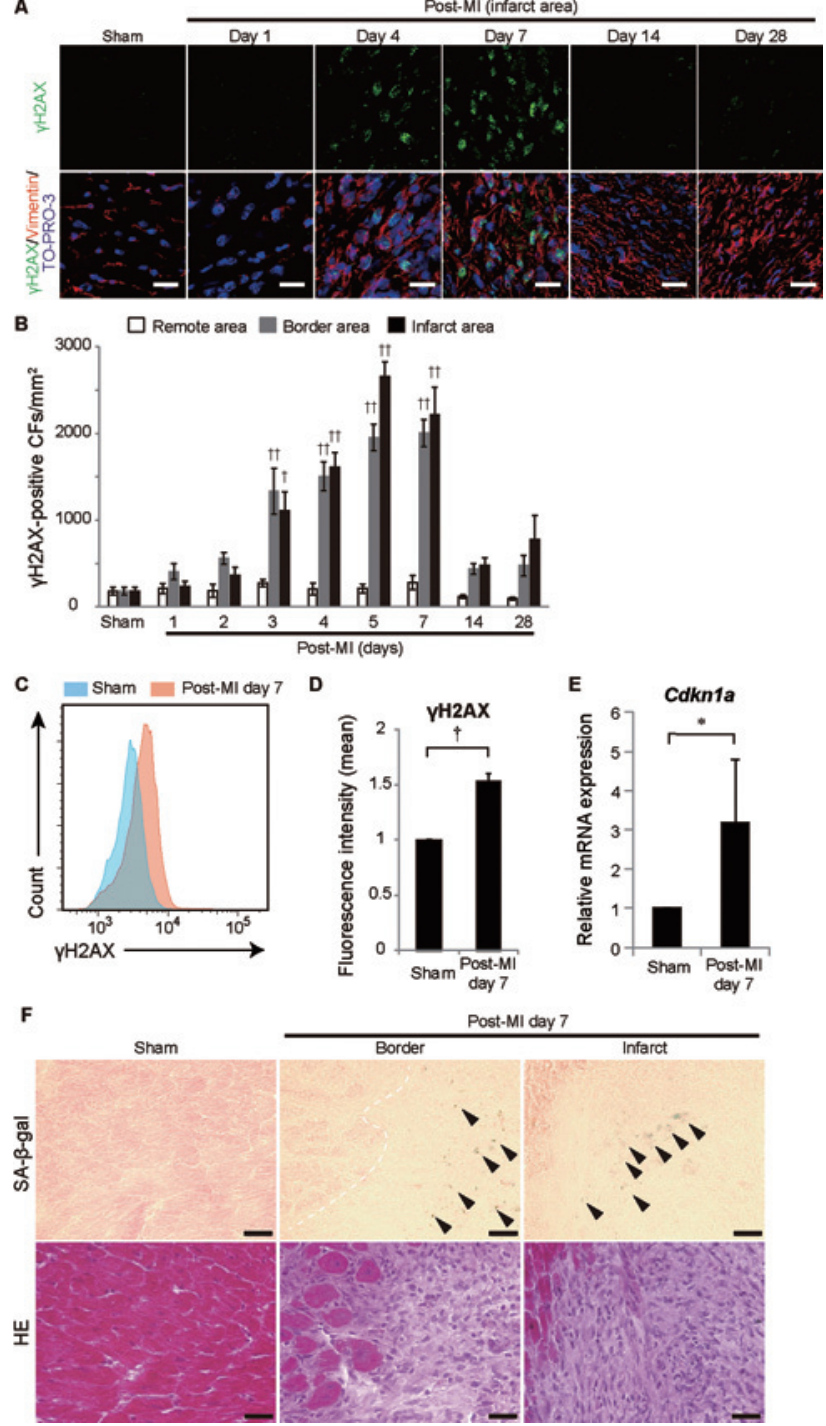

Figure 1. Activation of DNA damage response and cellular senescence in cardiac fibroblasts after myocardial infarction. A, B: The sections of heart tissue from sham- and MI-operated mice were immunostained for phosphorylated $\mathrm{H} 2 \mathrm{AX}(\gamma \mathrm{H} 2 \mathrm{AX}$, green). Vimentin (red) was used to label cardiac fibroblasts (CFs). Nuclei were stained with TO-PRO-3 (blue). Representative images in the infarct area (A, scale bar, $20 \mu \mathrm{m})$. The number of $\gamma \mathrm{H}_{2} \mathrm{AX}^{+}$vimentin ${ }^{+}$cells in the remote, border and infarct area were counted at the indicated time points (B, sham; $n=7$, Post-MI day $7 ; n=6$, Post-MI day $1,2,3,4,5,14,28 ; n=5$, respectively). The number of $\gamma \mathrm{H}_{2} \mathrm{AX}^{+}$vimentin ${ }^{+}$cells was counted from the randomly selected 5 fields. C, D: Non-cardiomyocytes were isolated from the hearts of sham- and MIoperated mice at day 7 after MI, and the fluorescent intensity of $\gamma \mathrm{H} 2 \mathrm{AX}$ in CD $31^{-} \mathrm{CD} 45^{-} \mathrm{CFs}$ was analyzed by flow cytometry. A representative histogram of $\gamma \mathrm{H} 2 \mathrm{AX}$ positivity in $\mathrm{CD} 31^{-} \mathrm{CD} 45^{-}$cells from the heart of shamand MI-operated mice (C, Sham: blue, MI: red). The difference in the fluorescent intensity was compared by the mean fluorescent intensity ( $n=8$ each). Statistical significance was determined by Student's $t$-test. $† P<$ 0.01 versus Sham. E: CD31-CD45-Thy $1^{+}$and/or CD31-CD45-PDGFR $\alpha^{+}$ CFs were isolated from non-cardiomyocytes by fluorescence activated cell sorting. The expression level of Cdknla gene in CFs was analyzed by quantitative real-time PCR ( $n=5$ each). Statistical significance was determined by the Mann-Whitney $U$-test. ${ }^{*} P<0.05$ versus Sham. F: The sections of heart tissue were stained with SA- $\beta$-gal (upper panels) and haematoxylin and eosin (lower panels) at day 7 after MI. Arrowheads indicate SA- $\beta$-gal positive cells. Scale bar, $25 \mu \mathrm{m}$. 
A

Sham

Post-MI day 4

Post-MI day 7
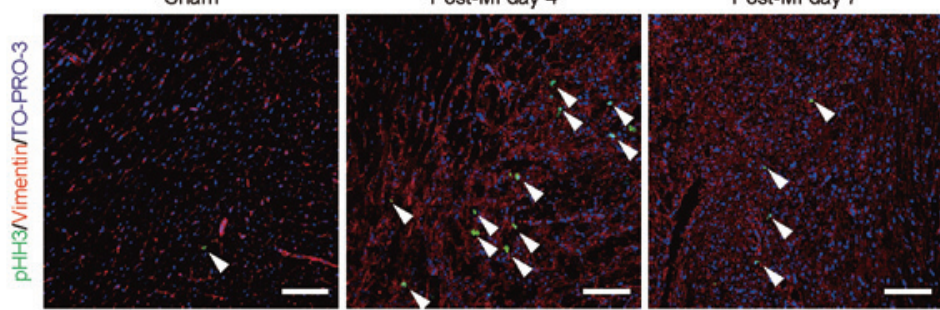

B

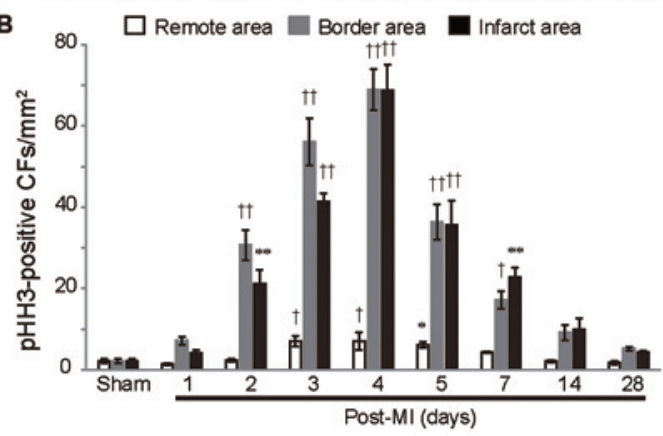

C

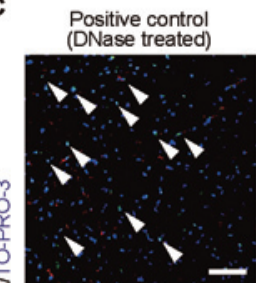

Post-MI day 4
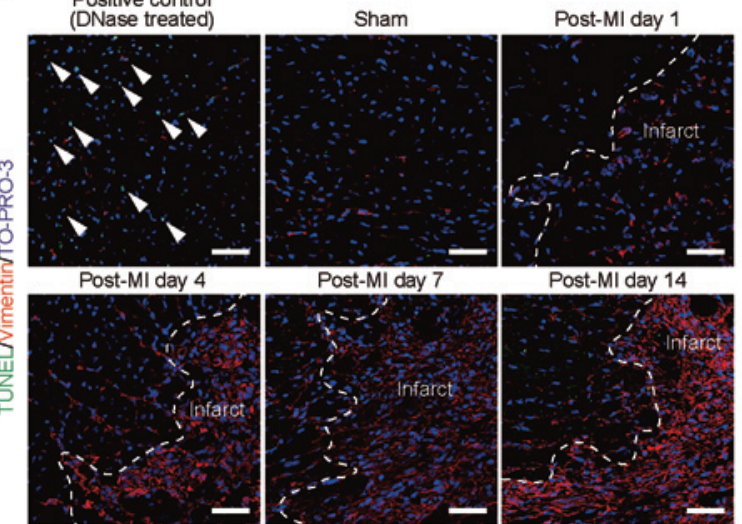

Post-MI day 7
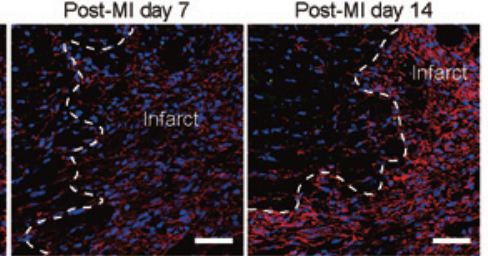

D

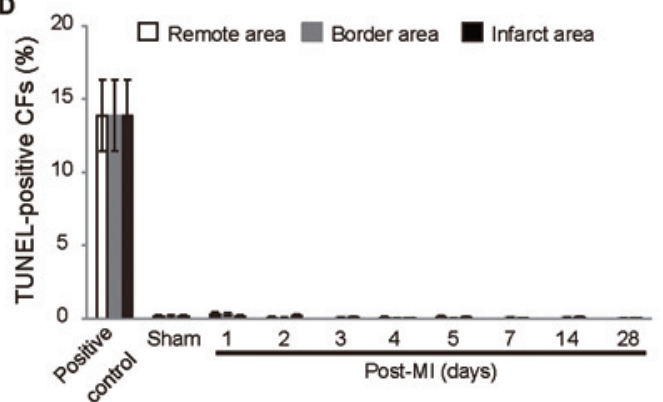

Figure 2. Proliferation of cardiac fibroblasts after myocardial infarction. A, B: The sections of heart tissue were immunostained for phosphorylated Histone $\mathrm{H} 3$ ( $\mathrm{pHH}$, green), vimentin (red) and TO-PRO-3 (blue). Representative images in the infarct area (A, scale bar, $100 \mu \mathrm{m})$. Arrowheads indicate $\mathrm{pHH} 3^{+}$vimentin ${ }^{+}$cells. The number of pHH3 ${ }^{+}$vimentin ${ }^{+}$CFs was counted at the indicated time points (B, sham; $n=7$, Post-MI day $7 ; n=6$, Post-MI day $1,2,3,4,5,14,28 ; n=5$, respectively). Statistical significance was determined by Dunnett test. $* P<0.05 ; \dagger P<0.01 ; * * P<0.001 ; \dagger \dagger P<$ 0.0001 versus Sham. C, D: The sections of heart tissue were stained for TdT-mediated dUTP nick end labeling (TUNEL, green), vimentin (red), and TO-PRO-3 (blue). DNase-treated (DNase I, 10 Kunitz units $/ \mathrm{mL}$ ) section was used as a positive control. Representative images in the border area $(\mathbf{C}$, scale bar, $50 \mu \mathrm{m})$. Arrowheads indicate TU$\mathrm{NEL}^{+}$cells. The percentage of TUNEL ${ }^{+} \mathrm{CFs}$ in remote, border and infarct area was calculated at the indicated time points (D, $n=4$ each). Column and error bars show mean and s.e.m., respectively. 
A
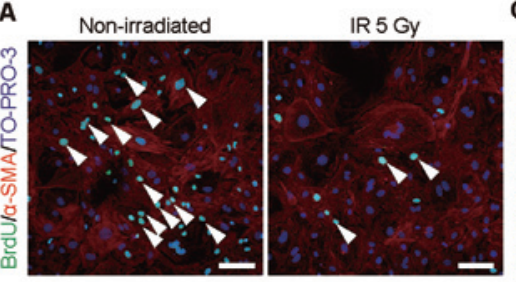

C
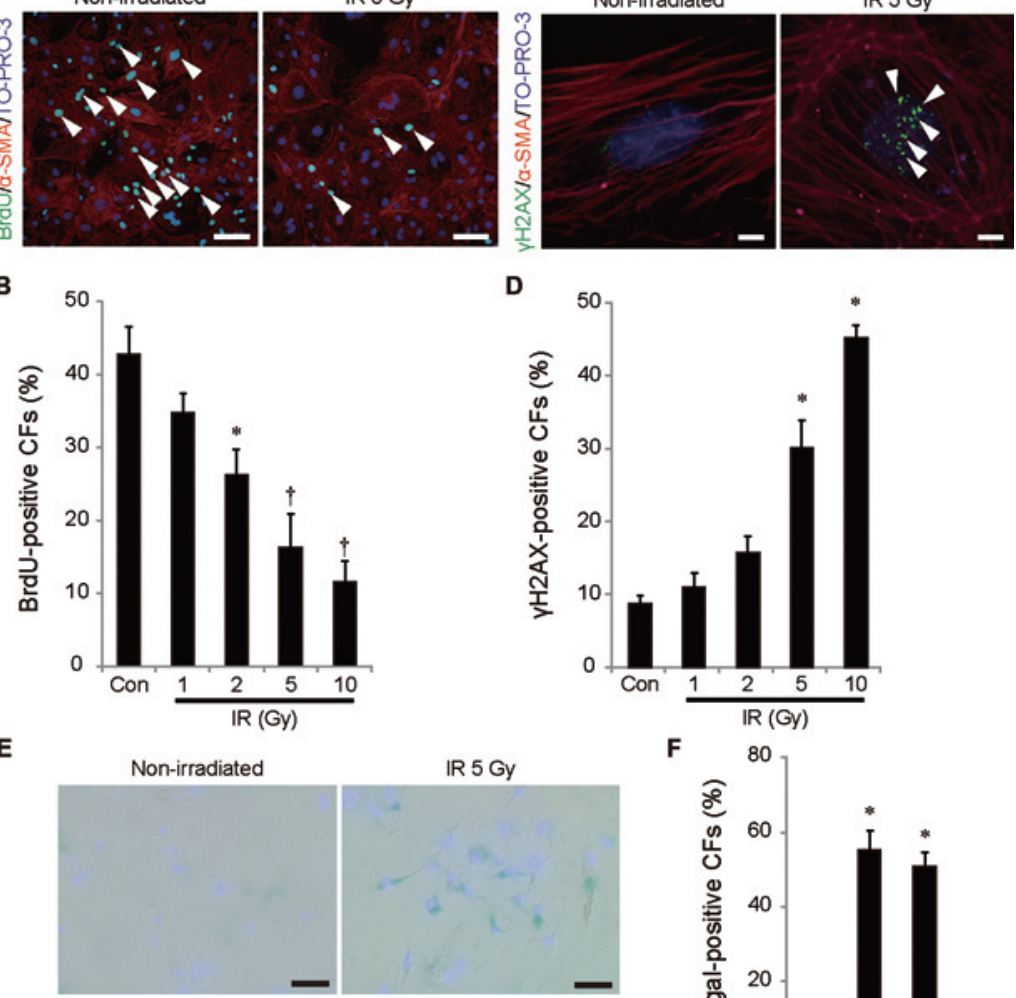

$\mathbf{F}$
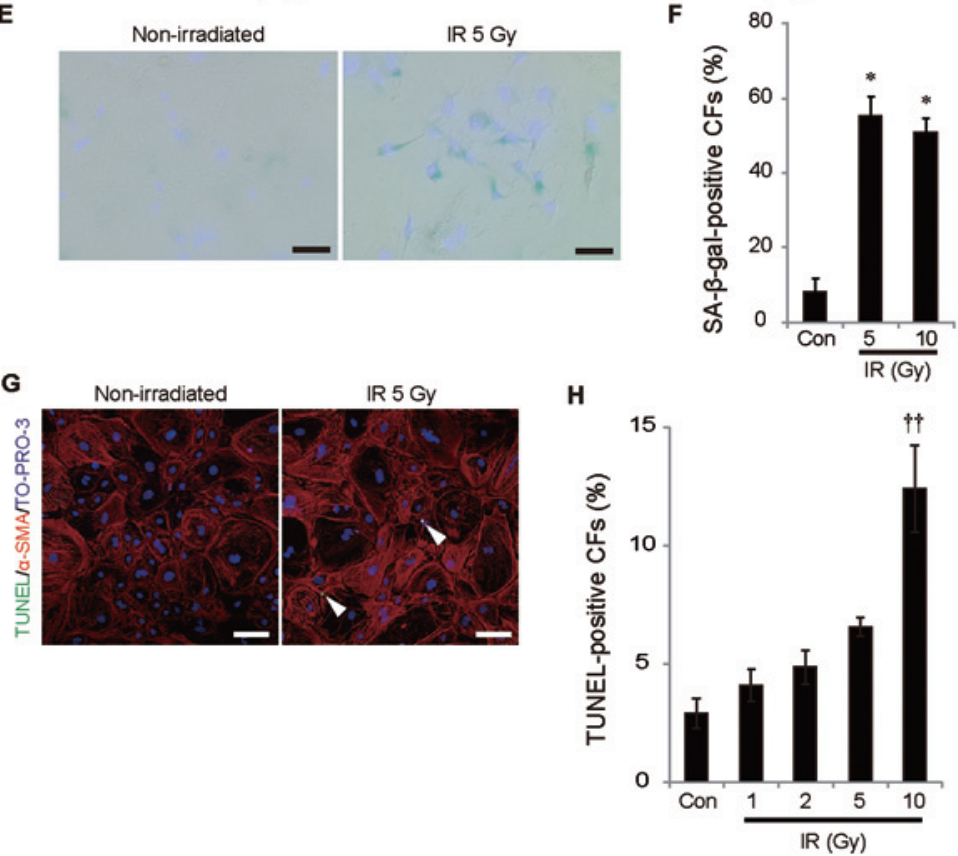

Figure 3. Establishment of an in vitro model of stress-induced senescence in CFs. A, B: Cultured CFs were irradiated (IR) at various dose and labeled by 5-bromo-2'-deoxyuridine (BrdU) $(10 \mu \mathrm{M})$ for the last 24 hours. Cells were then immunostained for BrdU (green), $\alpha$-smooth muscle actin ( $\alpha$-SMA, red) and TO-PRO-3 (blue). $\alpha$-SMA was used to label CFs. Representative images at the dose of 0 and $5 \mathrm{~Gy}(\mathbf{A}$, scale bar, $100 \mu \mathrm{m})$. Arrowheads indicate $\mathrm{BrdU}^{+} \alpha-\mathrm{SMA}^{+}$cells. The percentage of $\mathrm{BrdU}^{+}$cells within $\alpha-\mathrm{SMA}^{+}$cells was calculated at each dose (B, $n=4$ each). C, D: Cultured CFs were irradiated at various dose and immunostained for $\gamma \mathrm{H} 2 \mathrm{AX}$ (green), $\alpha$-SMA (red) and TO-PRO-3 (blue). Representative images at the dose of 0 and $5 \mathrm{~Gy}(\mathbf{C}$, scale bar, $5 \mu \mathrm{m})$. Arrowheads indicate $\gamma \mathrm{H} 2 \mathrm{AX}$ foci and the cells with more than 3 foci were defined as $\gamma \mathrm{H} 2 \mathrm{AX}^{+}$cell. The percentage of $\gamma \mathrm{H} 2 \mathrm{AX}^{+}$cells within $\alpha$-SMA ${ }^{+}$cells was calculated at each dose (D, $n=4$ each). E, F: Cultured CFs were irradiated and stained with SA- $\beta$-gal. Representative images at 5 Gy $(\mathbf{E}$, scale bar, $50 \mu \mathrm{m})$. The percentage of cells stained in blue was calculated (F, $n=4$ each). G, H: Cultured CFs were irradiated (IR) at various dose and stained for TUNEL (green), $\alpha$-SMA (red) and TO-PRO-3 (blue) at 48 hours after irradiation. Representative images of non-irradiated control CFs and CFs irradiated at $5 \mathrm{~Gy}(\mathbf{G}$, scale bar, $100 \mu \mathrm{m})$. Arrowheads indicate TUNEL ${ }^{+}$CFs. The percentage of TU$\mathrm{NEL}^{+} \mathrm{CFs}(\mathbf{H}, n=5$ each). Statistical significance was determined by Dunnett test for $\mathbf{B}, \mathbf{D}, \mathbf{F}$, and $\mathbf{H}$. $* P<0.05 ; \dagger P<0.01 \dagger \dagger P<0.0001$ versus non-irradiated control (Con). Column and error bars show mean and s.e.m., respectively. 
A
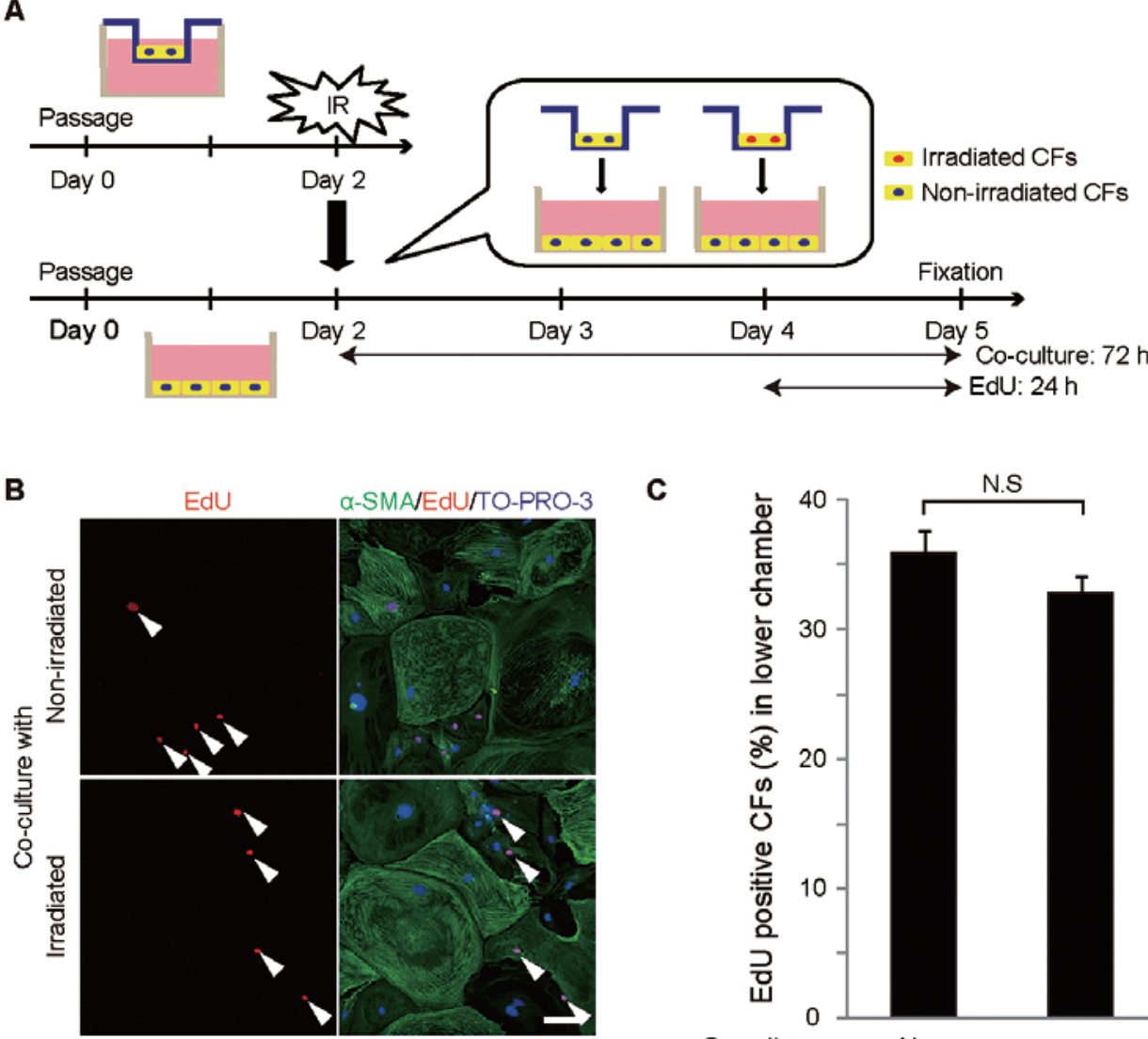

C

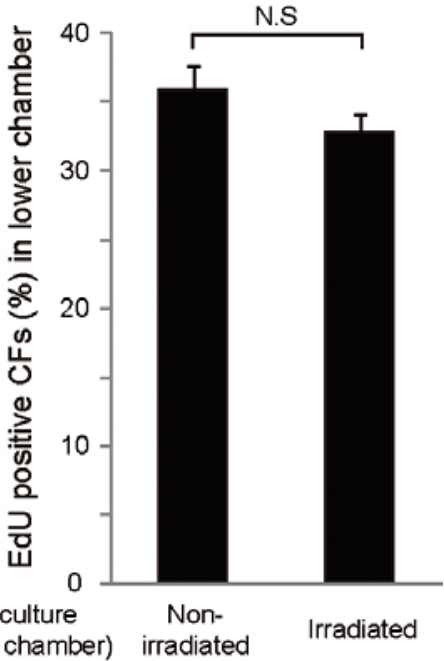

Figure 4. Senescent cardiac fibroblasts did not affect the proliferation of remote, non-senescent cardiac fibroblasts in a paracrine manner. A: Cardiac fibroblasts (CFs), cultured on the upper chamber, were irradiated at the dose of 0 (non-irradiated) or 5 Gy and transferred on the lower chamber, where non-irradiated CFs had been cultured. Co-culturing was continued for 72 hours. Proliferating cells were labeled by 5-ethynyl-2'-deoxyuridine $(E d U, 10 \mu \mathrm{M})$ for the last 24 hours and stained with EdU. B, C: The CFs in the lower chamber were stained with 5-ethynyl-2'-deoxyuridine (EdU, red), $\alpha$-SMA (green) and TO-PRO-3 (blue). Representative images (B, scale bar, $100 \mu \mathrm{m})$. Arrowheads indicate $\mathrm{EdU}^{+} \alpha-\mathrm{SMA}^{+}$cells. The fraction of EdU ${ }^{+}$cells within $\alpha-\mathrm{SMA}^{+}$cells was calculated $(\mathbf{C}, n=4$ each). Statistical significance was determined by Student's $t$-test. N.S indicates not significant.

phenomenon is also reported in the wound healing process after skin damage. ${ }^{27)}$ Taken together, senescence of fibroblasts seems to play a key role in tissue fibrosis through regulating the activation and proliferation of fibroblasts.

Cellular senescence is considered one of the antitumor mechanisms by which cells with extensive DNA damage no longer proliferate. ${ }^{10)}$ Senescent cells are not only stunned, non-proliferating cells but also affect their surrounding microenvironments through changing their gene expression patterns. This characteristic change in the gene expression pattern is called senescent-associated secretory phenotype (SASP). Through acquisition of SASP, senescent cells modify the disease processes by changing the cell-cell adhesion, secreting various kinds of cytokines such as IL-1 $\alpha$, IL-6, IL-8, and transforming growth factor (TGF)- $\beta{ }^{28)}$ In the present study, we examined the noncell autonomous effect of senescent CFs using Boyden chamber system and co-culture system. Interestingly, senescent CFs suppressed the proliferation of non-senescent CFs only when they were co-cultured, suggesting that contact-dependent mechanism is involved in the non-cell autonomous effect of senescent CFs. The precise mechanism by which senescent CFs suppress the proliferation of surrounding cells needs further investigation in the future study.

In the present study, we showed the detrimental effect of Atm gene deletion on cardiac fibrosis at the acute phase but not on the cardiac function and cardiac fibrosis at the chronic phase. This result is in clear contrast with the report from Daniel et al. showing that ATM deficiency exacerbates cardiac dysfunction as well as cardiac fibrosis after MI even at the chronic phase. ${ }^{29)}$ Given that the mortality rate was higher $(44 \%-61 \%)$ in the previous report compared to the present study $(\sim 80 \%)$, it is suggested that the difference in the infarct size may explain the discrepancy between the results in both studies.

From clinical perspectives, adequate induction of cellular senescence in CFs may become a novel therapeutic strategy for preventing adverse cardiac remodeling after MI. Suppression of premature senescence in CFs by ge- 


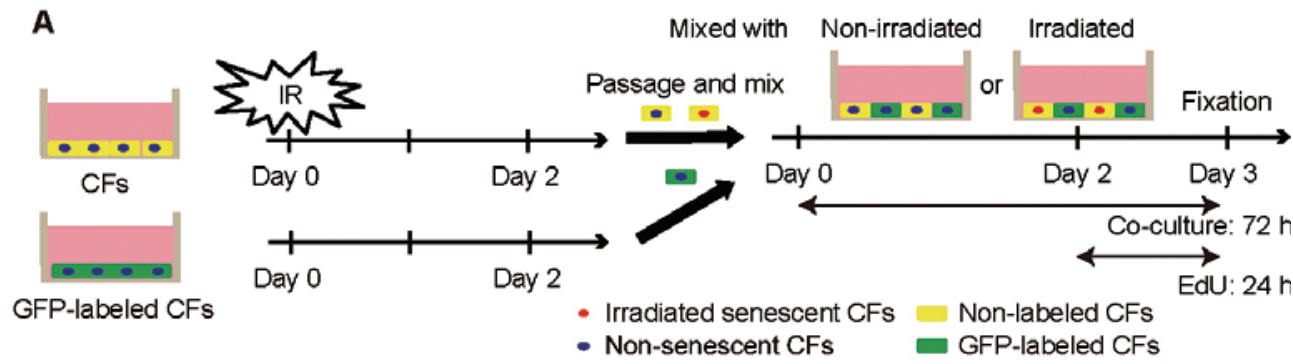

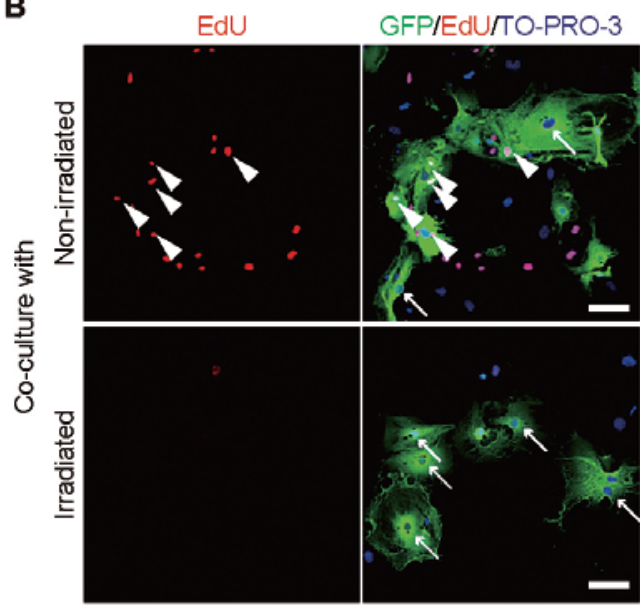

D
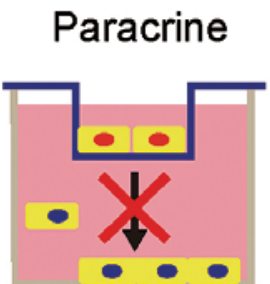

C

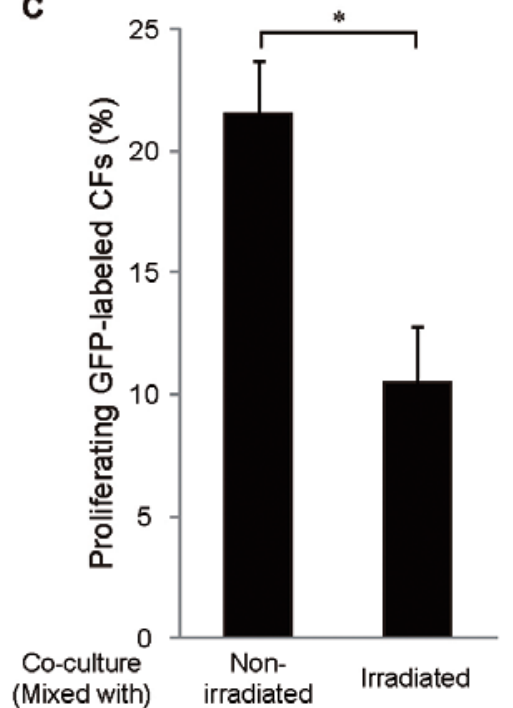

\section{Senescent CFs Non-senescent CFs}

Figure 5. Senescent cardiac fibroblasts suppressed the proliferation of surrounding non-senescent cardiac fibroblasts in a juxtacrine manner. A: Non-labeled CFs were irradiated at the dose of 0 (non-irradiated) or 5 Gy and mixed with green fluorescent protein (GFP) -labeled CFs, which were cultured independently. Non-labeled CFs were mixed and cultured with GFP-labeled CFs for 72 hours, and the proliferating cells were labeled by EdU $(10 \mu \mathrm{M})$ for the last 24 hours and stained with EdU. B, C: The co-culture of GFP-labeled CFs and non-labeled CFs were stained with EdU (red), GFP (green) and TO-PRO-3 (blue). Representative images $\left(\mathbf{B}\right.$, scale bar, $100 \mu \mathrm{m}$ ). The fraction of EdU $\mathrm{C}^{+}$cells within $\mathrm{GFP}^{+}$cells was calculated $\left(\mathbf{C}, n=4\right.$ each). Arrowheads indicate $\mathrm{EdU}^{+} \mathrm{GFP}^{+}$cells. Arrows indicate $\mathrm{EdU}^{-} \mathrm{GFP}^{+}$ cells. Statistical significance was determined by Student's t-test. $* P<0.05$ versus Non-irradiated. D: Senescent CFs suppressed the proliferation of the surrounding non-senescent CFs in a cell-cell contact-dependent manner.

netic ablation of p53 and p16 $6^{\mathrm{INK} 4}$, which play a key role in cellular senescence, leads to aggravated cardiac fibrosis in pressure overload-induced heart failure model mice. ${ }^{30)}$ In contrast, induction of premature senescence in $\mathrm{CFs}$ by gene transfer of $\mathrm{CCN}$ family member $1^{27,30)}$ limits cardiac fibrosis and improves cardiac function. In the present study, we also found that the suppression of DDR and premature senescence by Atm gene deletion exacerbated cardiac fibrosis after MI. A report from other groups also show that administration of RITA (reactivation of p53 and induction of tumor cell apoptosis), a stabilizer of a major DDR effector protein p53, attenuates cardiac fibrosis in a mouse model of ischemia-reperfusion cardiac injury. ${ }^{31}$ Taken together, these findings suggest that cellular senescence in CFs is necessary and sufficient to limit cardiac fibrosis, irrespective of the etiology of heart failure, and 
A

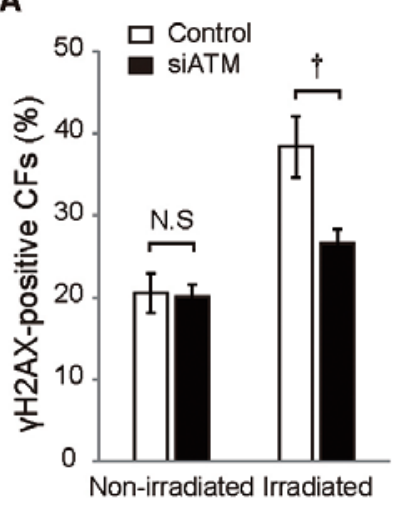

B

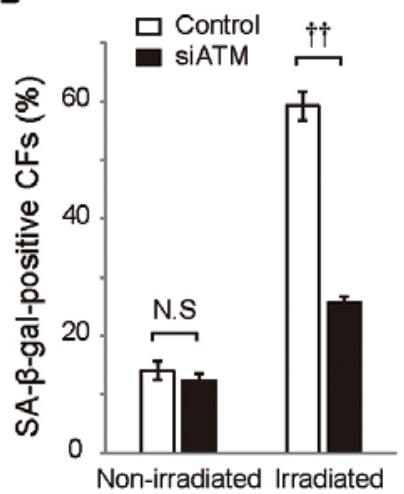

$\mathbf{C}$

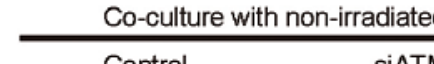

Co-culture with irradiated
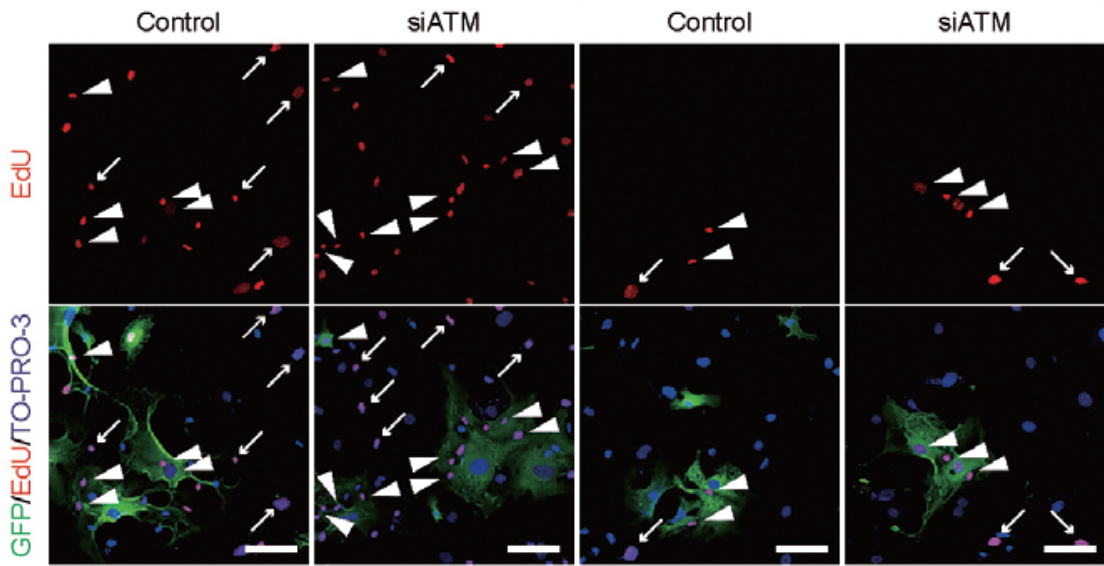

D

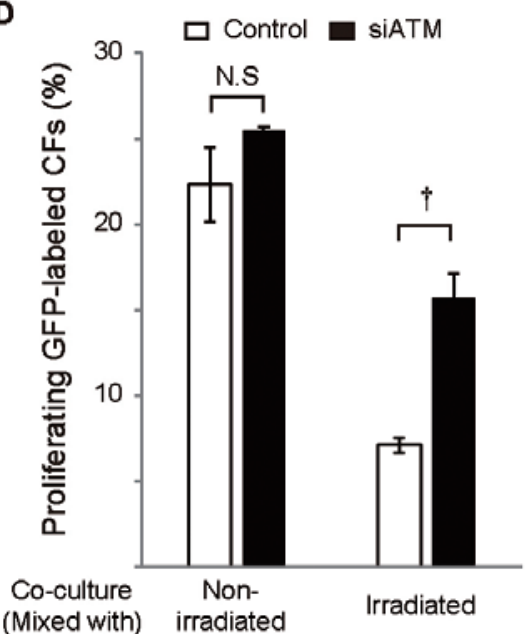

Figure 6. ATM mediates the juxtacrine effect of senescent cardiac fibroblasts on the proliferation of surrounding non-senescent cardiac fibroblasts. A: After siRNA transfection, cultured CFs were irradiated at $5 \mathrm{~Gy}$ and immunostained for $\gamma \mathrm{H} 2 \mathrm{AX}, \alpha$-SMA and TO-PRO-3. The percentage of $\gamma \mathrm{H} 2 \mathrm{AX}^{+}$cells was calculated ( $n=4$ each). The cells with more than 3 foci were defined as $\gamma \mathrm{H} 2 \mathrm{AX}^{+}$. (Control). B: Irradiated CFs were stained with senescence-associated beta-galactosidase (SA- $\beta$-gal). The percentage of cells stained in blue was calculated ( $n=5$ each). C, D: Non-labeled CFs were transfected with siRNA against Atm before irradiation and mixed-cultured as described in Figure 5A. Representative images of the stained CFs stained with EdU (red), GFP (green) and TO-PRO-3 (blue) (C, scale bar, 100 $\mu \mathrm{m})$. Arrowheads indicate $\mathrm{EdU}^{+} \mathrm{GFP}^{+}$cells and arrows indicate $\mathrm{EdU}^{+} \mathrm{GFP}^{-}$cells. The fraction of $\mathrm{EdU}^{+}$ cells within $\mathrm{GFP}^{+}$cells were calculated $(\mathbf{D}, n=6$ each). Statistical significance was determined by Tukey-Kramer HSD test. $\uparrow P<0.01, \dagger \dagger P<0.0001$ versus negative control (Control). N.S indicates not significant. Column and error bars show mean and s.e.m., respectively. 
A

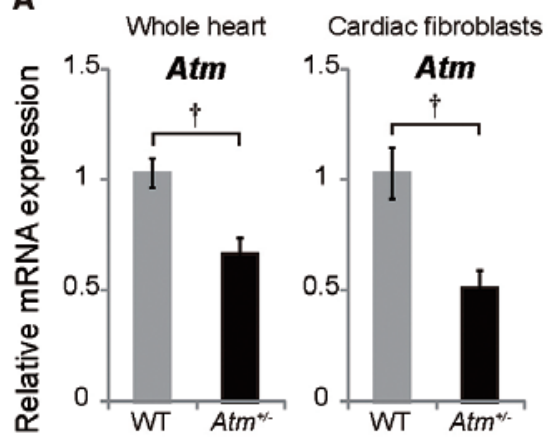

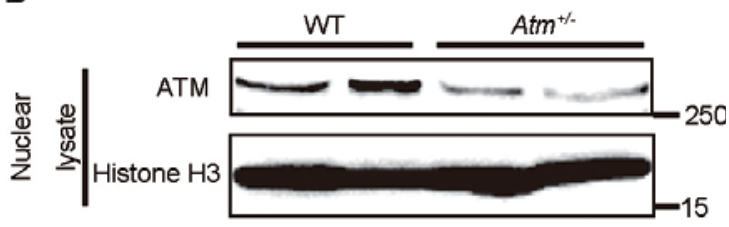

C

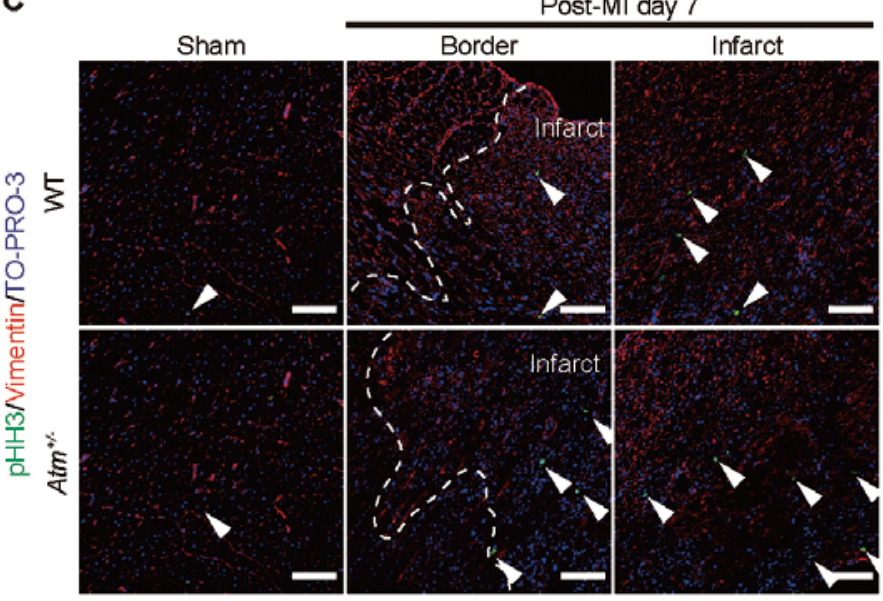

D

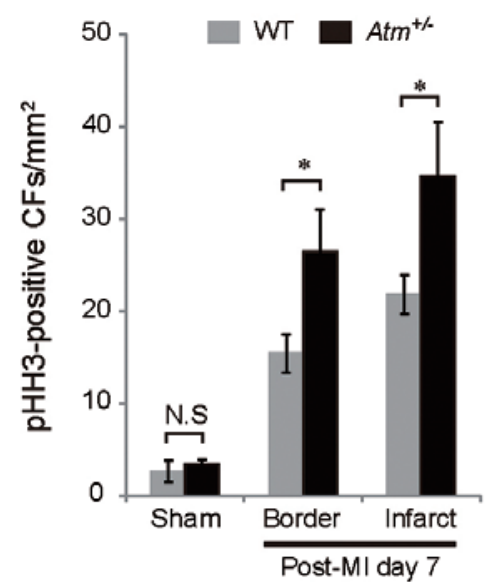

E

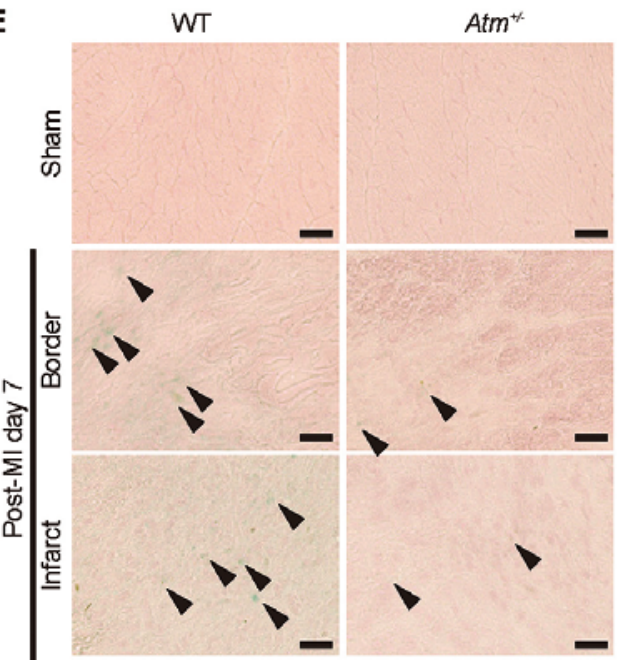

Figure 7. Cardiac fibroblast remained proliferative after myocardial infarction in ATM heterozygous mice. A: The expression levels of Atm in the whole heart tissue (left panel, $n=4$ each) and sorted cardiac fibroblasts (right panel, WT; $n=4, \mathrm{Atm}^{+-} ; n=6$ ) were measured by quantitative real-time PCR. B: The expression level of nuclear ATM protein was assessed by western blotting. Histone $\mathrm{H} 3$ was used as loading control. Statistical significance was determined by Student's t-test. $\dagger P<0.01$ versus WT. Column and error bars show mean and s.e.m., respectively. C, D: The sections of heart tissue from Sham- and MI-operated WT and $\mathrm{Atm}^{+/-}$mice were stained for phosphorylated Histone H3 ( $\mathrm{pHH} 3$, green), vimentin (red), and TO-PRO-3 (blue) at day 7 after MI. Arrowheads indicate $\mathrm{pHH}^{+}{ }^{+}$vimentin ${ }^{+}$cells. Representative images $(\mathbf{C}$, scale bar, $100 \mu \mathrm{m})$. The number of $\mathrm{pHH}^{+}{ }^{+}$vimentin ${ }^{+}$cells in the border and infarct area was counted (D, sham; $n=3$ each, post-MI day $7 ; n=7-8$ ). (E) The sections of heart tissue were stained with senescenceassociated beta-galactosidase (SA- $\beta$-gal) at day 7 after MI. Arrowheads indicate SA- $\beta$-gal positive cells. Scale bar, 25 $\mu \mathrm{m}$. Statistical significance was determined by Student's $t$-test. $* P<0.05 ; \uparrow P<0.01$ versus WT mice. N.S indicates not significant. Column and error bars show mean and s.e.m., respectively. 


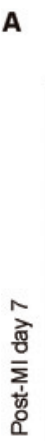
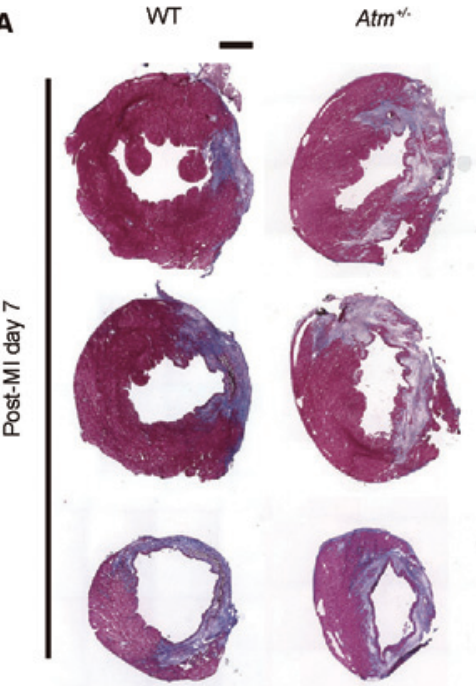

B

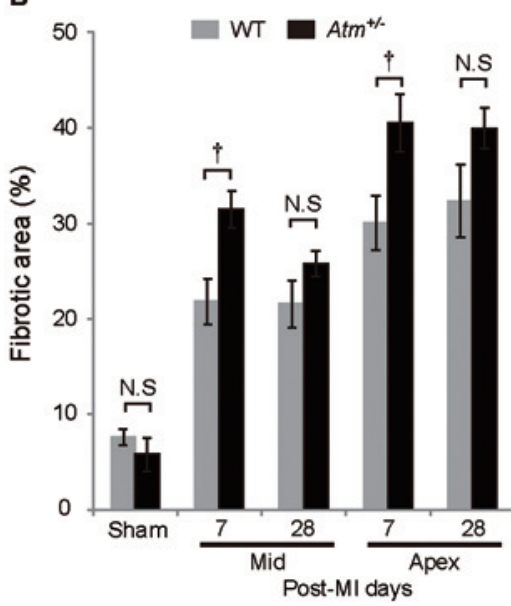

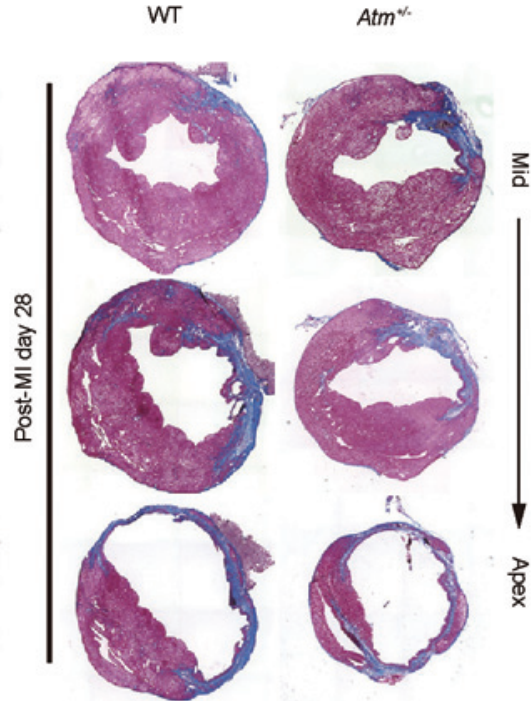

C
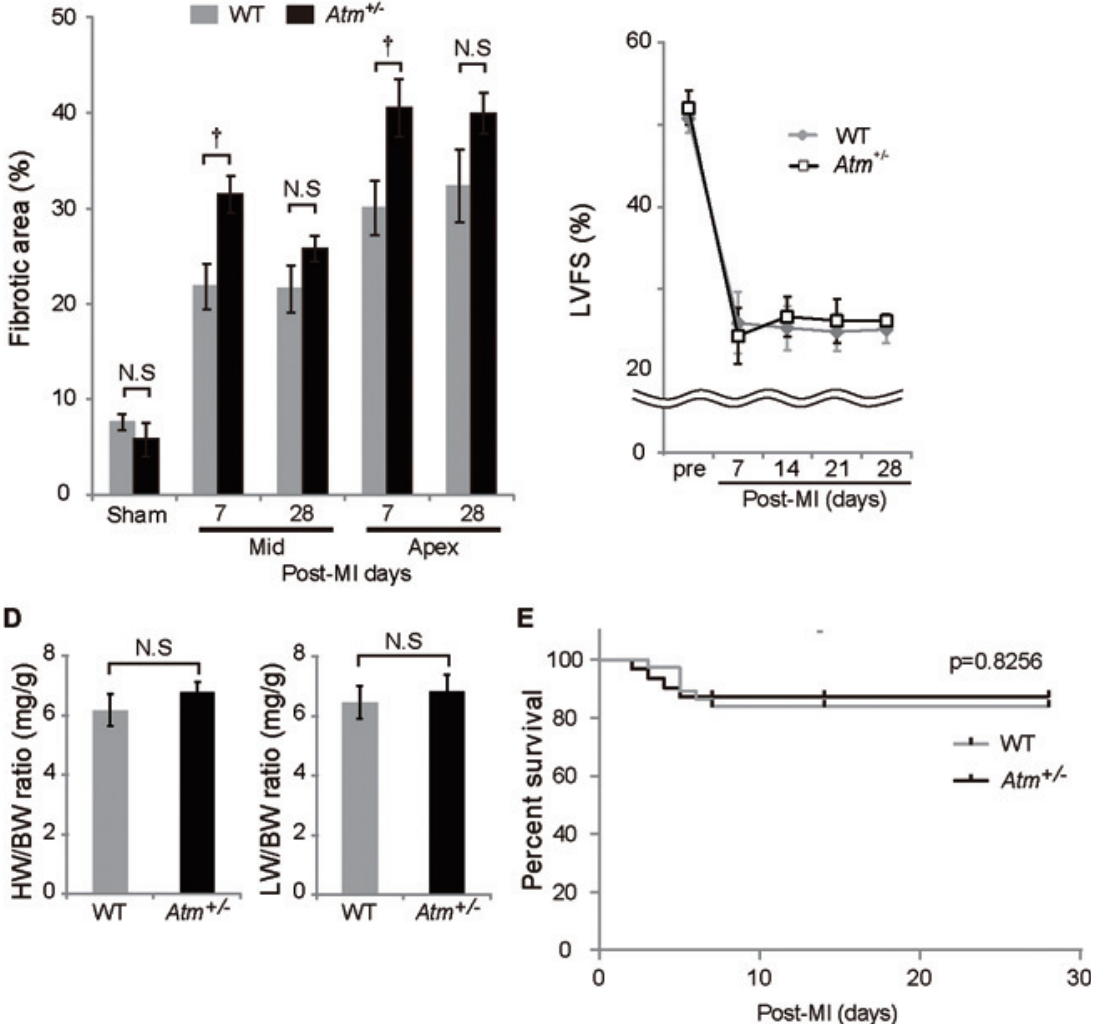

Figure 8. Cardiac fibrosis was exacerbated at the acute phase after myocardial infarction in ATM heterozygous mice. A, B: The sections of heart tissue were stained with Masson's trichrome staining at day 7 and 28 after MI. Representative images (A, scale bar, $1 \mathrm{~mm}$ ). The percentage of fibrotic area was calculated as the ratio of the fibrotic area to the total cross-sectional area of left ventricle (B, sham; $n=3$ each, post-MI day 7; $n=5$ each, post-MI day $28 ; n=5$, 4, respectively). C: The left ventricular (LV) function of MI-operated WT and $\mathrm{Atm}^{+/-}$mice was assessed by echocardiogram at the indicated time points after MI $(n=4$ each). LVFS, LV fractional shortening. D: Heart, lung, and body weight of myocardial infarction (MI) -operated WT and $\mathrm{Atm}^{+/-}$mice were weighed at day 7 after MI. The heart weight or lung weight per body weight were calculated ( $n=4$ each). Statistical significance was determined by Student's t-test. N.S; not significant. Column and error bars show mean and s.e.m., respectively. Statistical significance was determined by Student's $t$-test for B, C, and D. $\dagger P<0.01$ versus WT mice. N.S indicates not significant. Column and error bars show mean and s.e.m., respectively. E: Survival curve of WT and $\mathrm{Atm}^{+/-}$mice after MI operation $(n=37,31$, respectively). Statistical significance was determined by Wilcoxon test. 


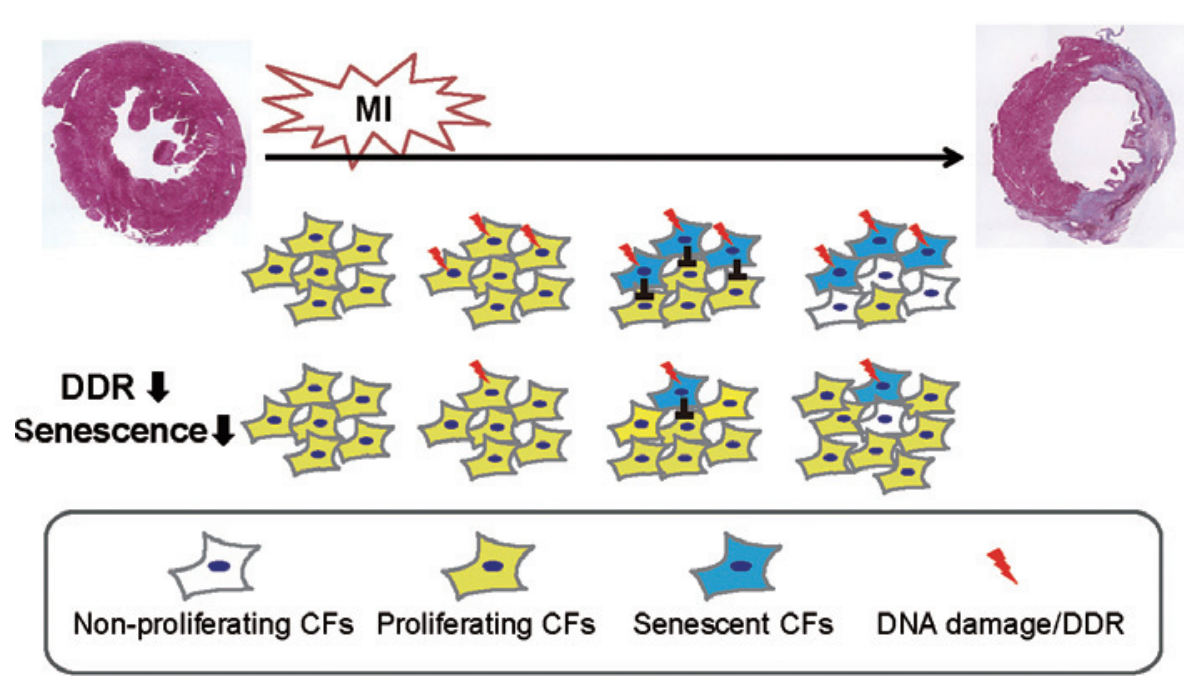

Figure 9. The proposed mechanism by which senescent $\mathrm{CFs}$ limit cardiac fibrosis after myocardial infarction. The number of CFs with active DDR increase after MI. DDR become persistent in some of the CFs, leading to cellular senescence of CFs. Senescent CFs suppress the proliferation of surrounding CFs in a cellcell contact-dependent manner, at least at the acute phase after MI. ATM plays essential role in this process and genetic deletion of ATM exacerbates cardiac fibrosis after MI.

that the DDR signaling pathway may become a potential therapeutic target for preventing adverse cardiac remodeling. Our results highlight the role of DDR activation and cellular senescence in 'fine-tuning' of the CF proliferation and delivering appropriate cardiac fibrosis after MI (Figure 9). Given that insufficient activation and proliferation of CFs lead to cardiac rupture, ${ }^{32)}$ proper timing and the extent for inducing cellular senescence in CFs should be investigated before clinical applications.

\section{Acknowledgments}

We thank M. Shimizu, H. Taniwaki, A. Matsunaga, Y. Ueda, and K. Kawaguchi for their technical support. This study was supported by Center for Medical Research and Education, Graduate School of Medicine, Osaka University.

\section{Disclosure}

Conflicts of interest: The authors declare no competing financial interests.

\section{References}

1. Ambrosy AP, Fonarow GC, Butler J, et al. The global health and economic burden of hospitalizations for heart failure: lessons learned from hospitalized heart failure registries. J Am Coll Cardiol 2014; 63: 1123-33.

2. Shinde AV, Frangogiannis NG. Fibroblasts in myocardial infarction: a role in inflammation and repair. J Mol Cell Cardiol 2014; 70: 74-82.

3. Fan D, Takawale A, Lee J, Kassiri Z. Cardiac fibroblasts, fibrosis and extracellular matrix remodeling in heart disease. Fibrogenesis Tissue Repair 2012; 5: 15.

4. Talman V, Ruskoaho H. Cardiac fibrosis in myocardial infarction-from repair and remodeling to regeneration. Cell Tissue Res 2016; 365: 563-81.

5. Travers JG, Kamal FA, Robbins J, Yutzey KE, Blaxall BC. Cardiac fibrosis: the fibroblast awakens. Circ Res 2016; 118: 102140.

6. Gourdie RG, Dimmeler S, Kohl P. Novel therapeutic strategies targeting fibroblasts and fibrosis in heart disease. Nat Rev Drug Discov 2016; 15: 620-38.

7. Tian HP, Sun YH, He L, Yi YF, Gao X, Xu DL. Single-stranded DNA-binding protein 1 abrogates cardiac fibroblast proliferation and collagen expression induced by angiotensin II. Int Heart J 2018; 59: 1398-408

8. Shiloh Y. The ATM-mediated DNA-damage response: taking shape. Trends Biochem Sci 2006; 31: 402-10.

9. Bensimon A, Aebersold R, Shiloh Y. Beyond ATM: the protein kinase landscape of the DNA damage response. FEBS Lett 2011; 585: 1625-39.

10. Campisi J, d'Adda di Fagagna F. Cellular senescence: when bad things happen to good cells. Nat Rev Mol Cell Biol 2007; 8: $729-40$.

11. Salama R, Sadaie M, Hoare M, Narita M. Cellular senescence and its effector programs. Genes Dev 2014; 28: 99-114.

12. Krizhanovsky V, Yon M, Dickins RA, et al. Senescence of activated stellate cells limits liver fibrosis. Cell 2008; 134: 657-67.

13. Lujambio A, Akkari L, Simon J, et al. Non-cell-autonomous tumor suppression by p53. Cell 2013; 153: 449-60.

14. Barlow C, Hirotsune S, Paylor R, et al. Atm-deficient mice: a paradigm of ataxia telangiectasia. Cell 1996; 86: 159-71.

15. Okabe M, Ikawa M, Kominami K, Nakanishi T, Nishimune Y. 'Green mice' as a source of ubiquitous green cells. FEBS Lett 1997; 407: 313-9.

16. Muthuramu I, Lox M, Jacobs F, De Geest B. Permanent ligation of the left anterior descending coronary artery in mice: a model of post-myocardial infarction remodelling and heart failure. J Vis Exp 2014; 2: e52206.

17. Nakagawa A, Naito AT, Sumida T, et al. Activation of endothelial beta-catenin signaling induces heart failure. Sci Rep 2016; 6: 25009.

18. Dimri GP, Lee X, Basile G, et al. A biomarker that identifies senescent human cells in culture and in aging skin in vivo. Proc Natl Acad Sci U S A 1995; 92: 9363-7. 
19. Fumagalli M, Rossiello F, Clerici M, et al. Telomeric DNA damage is irreparable and causes persistent DNA-damageresponse activation. Nat Cell Biol 2012; 14: 355-65.

20. Johmura Y, Shimada M, Misaki T, et al. Necessary and sufficient role for a mitosis skip in senescence induction. Mol Cell 2014; 55: 73-84.

21. Acosta JC, Banito A, Wuestefeld T, et al. A complex secretory program orchestrated by the inflammasome controls paracrine senescence. Nat Cell Biol 2013; 15: 978-90.

22. Hoare M, Ito Y, Kang TW, et al. NOTCH1 mediates a switch between two distinct secretomes during senescence. Nat Cell Biol 2016; 18: 979-92.

23. Kopplow K, Letschert K, Konig J, Walter B, Keppler D. Human hepatobiliary transport of organic anions analyzed by quadrupletransfected cells. Mol Pharmacol 2005; 68: 1031-8.

24. Mallette FA, Gaumont-Leclerc MF, Ferbeyre G. The DNA damage signaling pathway is a critical mediator of oncogeneinduced senescence. Genes Dev 2007; 21: 43-8.

25. Zhan H, Suzuki T, Aizawa K, Miyagawa K, Nagai R. Ataxia telangiectasia mutated (ATM)-mediated DNA damage response in oxidative stress-induced vascular endothelial cell senescence. J Biol Chem 2010; 285: 29662-70.
26. Higo T, Naito AT, Sumida T, et al. DNA single-strand breakinduced DNA damage response causes heart failure. Nat Commun 2017; 8: 15104.

27. Jun JI, Lau LF. The matricellular protein CCN1 induces fibroblast senescence and restricts fibrosis in cutaneous wound healing. Nat Cell Biol 2010; 12: 676-85.

28. Coppe JP, Desprez PY, Krtolica A, Campisi J. The senescenceassociated secretory phenotype: the dark side of tumor suppression. Annu Rev Pathol 2010; 5: 99-118.

29. Daniel LL, Scofield SL, Thrasher P, et al. Ataxia telangiectasiamutated kinase deficiency exacerbates left ventricular dysfunction and remodeling late after myocardial infarction. Am J Physiol Heart Circ Physiol 2016; 311: H445-52.

30. Meyer K, Hodwin B, Ramanujam D, Engelhardt S, Sarikas A. Essential role for premature senescence of myofibroblasts in myocardial fibrosis. J Am Coll Cardiol 2016; 67: 2018-28.

31. Ubil E, Duan J, Pillai IC, et al. Mesenchymal-endothelial transition contributes to cardiac neovascularization. Nature 2014; 514: 585-90.

32. Shimazaki M, Nakamura K, Kii I, et al. Periostin is essential for cardiac healing after acute myocardial infarction. J Exp Med 2008; 205: 295-303. 